\title{
Role of PKCtheta in macrophage-mediated immune response to Salmonella typhimurium infection in mice
}

\author{
Christa Pfeifhofer-Obermair ${ }^{1,2+}$, Karin Albrecht-Schgoer ${ }^{1 \dagger}$, Sebastian Peer ${ }^{1}$, Manfred Nairz ${ }^{2}$, Kerstin Siegmund ${ }^{1}$, \\ Victoria Klepsch¹, David Haschka², Nikolaus Thuille', Natascha Hermann-Kleiter ${ }^{1}$, Thomas Gruber ${ }^{1}$, \\ Günter Weiss ${ }^{2}$ and Gottfried Baier ${ }^{1 *}(\mathbb{D})$
}

\begin{abstract}
Background: The serine/threonine protein kinase C (PKC) theta has been firmly implicated in T cell-mediated immunity. Because its role in macrophages has remained undefined, we employed PKCtheta-deficient $\left(\right.$ PKCtheta $\left.^{-/-}\right)$ mice in order to investigate if PKCtheta plays a role in macrophage-mediated immune responses during bacterial infections.

Results: Our results demonstrate that PKCtheta plays an important role in host defense against the Gram-negative, intracellular bacterium Salmonella typhimurium, as reflected both by markedly decreased survival and a significantly enhanced number of bacteria in spleen and liver of PKCtheta ${ }^{-1}$ mice, when compared to wild-type mice. Of note, albeit macrophages do not express detectable PKCtheta, PKCtheta mRNA expression was found to be profoundly upregulated during the first hours of lipopolysaccharide (LPS)/interferon-gamma (IFNgamma)-, but not IL-4-mediated cell polarization conditions in vitro. Mechanistically, despite expressing normal levels of classically activated macrophage (CAM) markers, PKCtheta-deficient CAMs expressed significantly higher levels of the anti-inflammatory cytokine IL-10 in vivo and in vitro when challenged with S. typhimurium or LPS/IFNgamma. Neutralization of IL-10 recovered immune control to $S$. typhimurium infection in PKCtheta-deficient macrophages.

Conclusions: Taken together, our data provide genetic evidence that PKCtheta promotes a potent pro-inflammatory CAM phenotype that is instrumental to mounting protective anti-bacterial immunity. Mechanistically, PKCtheta exerts a host-protective role against S. typhimurium infection, and acts as an essential link between TLR4/IFNgammaR signaling and selective suppression of the anti-inflammatory cytokine IL-10 at the onset of CAM differentiation in the course of a bacterial infection.
\end{abstract}

Keywords: Salmonella typhimurium, Protein kinase C theta, Innate immunity, Macrophage polarization, IL-10

\section{Background}

Macrophages $(\mathrm{M} \phi)$ play important roles in inflammatory and infectious diseases. $\mathrm{M} \phi$ polarization phenotypes, however, are heterogeneous and profoundly affected by local factors within the microenvironment. Microbial stimuli such as lipopolysaccharide (LPS), interleukin-1beta (IL1beta), and cytokines secreted by Th1 lymphocytes, such as interferon-gamma (IFNgamma) induce a "classic" $\mathrm{\phi} \phi$

\footnotetext{
* Correspondence: gottfried.baier@i-med.ac.at

${ }^{\dagger}$ Equal contributors

${ }^{1}$ Department for Pharmacology and Genetics, Division of Translational Cell

Genetics, Peter Mayr Straße 1a, 6020 Innsbruck, Austria

Full list of author information is available at the end of the article
}

phenotype (CAM). Activated CAMs are pro-inflammatory $\mathrm{M} \phi$ and produce high levels of pro-inflammatory cytokines like tumor necrosis factor-alpha (TNF-alpha), IL-6, IL1beta, IL-23 and IL-12. CAMs are essential components of anti-microbial host defense and further characterized by production of reactive oxygen species and reactive nitrogen species during the promotion of a Th1-type driven response [1-3]. Because these pro-inflammatory CAMs may cause extensive tissue damage, their activation is tightly controlled. Indeed, CAMs are established to be involved in autoimmune diseases such as systemic lupus erythematosus and rheumatoid arthritis [4]. In contrast, Th2 lymphocyte 
cytokines such as IL-4 and IL-13 promote the alternatively activated $M \phi$ phenotype (AAM) that dampens the inflammatory state by producing anti-inflammatory mediators such as IL-10. Signature genes for AAMs are Arginase-1, chitinase-like molecules YM1 and YM2, and resistin-like molecule Fizz-1 (Relm-alpha, Retnla) [5, 6]. AAMs, in particular, produce high amounts of the anti-inflammatory cytokines IL-10 and Tumor growth factor beta (TGF $\beta$ ), thereby inhibiting pro-inflammatory immune responses. Thus, AAMs are involved in tissue remodelling [7] and tumor progression [8].

Protein kinase $\mathrm{C}$ (PKC) isotypes are members of the serine/threonine protein kinase subfamily, and play an important role in the regulation of a variety of cell functions. PKCtheta (PKCtheta), a member of the nPKC subfamily, is predominantly expressed in T cells. Most of our current knowledge about PKCtheta therefore arises from studies performed in T cells. We and others could show that PKCtheta plays a critical role in the NFkappaB, AP-1, and $\mathrm{Ca}^{2+} /$ NFAT pathways to activate e.g. the interleukin-2 cytokine promoter $[9,10]$. Madaro et al. demonstrated that PKCtheta has an immune cell intrinsic role in muscle tissue repair during muscular dystrophy inflammation [11]. More recently, $\mathrm{Ma}$ et al. reported a role for PKCtheta in cholesterol metabolism in human $\mathrm{M} \phi$ [12]. Because different PKCs, namely PKCalpha, PKCbeta, PKCdelta and PKCzeta, have been shown to have a critical role in $\mathrm{M} \phi$ antimicrobial immune responses [13-16], we investigated the functional role of PKCtheta in $M \phi$ biology. Here we demonstrate that the genetic ablation of PKCtheta expression in mice leads to exacerbation of disease progression and early death in Salmonella (S.) typhimurium infection.

\section{Results}

PKCtheta mRNA expression is selectively induced in LPS/ IFNgamma polarizing conditions

PKCtheta has been reported to regulate the expression of various genes in $\mathrm{T}$ cells [17]. To examine whether PKCtheta modulates $\mathrm{M} \phi$ polarization in pro- or antiinflammatory milieu conditions, we analysed mRNA expression patterns of PKCtheta in bone marrow-derived macrophages (BMDM) polarized with lipopolysaccharide (LPS)/interferon-gamma (IFNgamma) or with IL-4, respectively (Fig. 1a). For proper polarization, marker gene expression of iNOS (for CAM) and Fizz-1 (for AAM) was monitored (Fig. 1b $+\mathrm{c}$ ). As a remarkable result, PKCtheta expression was strongly induced in LPS/
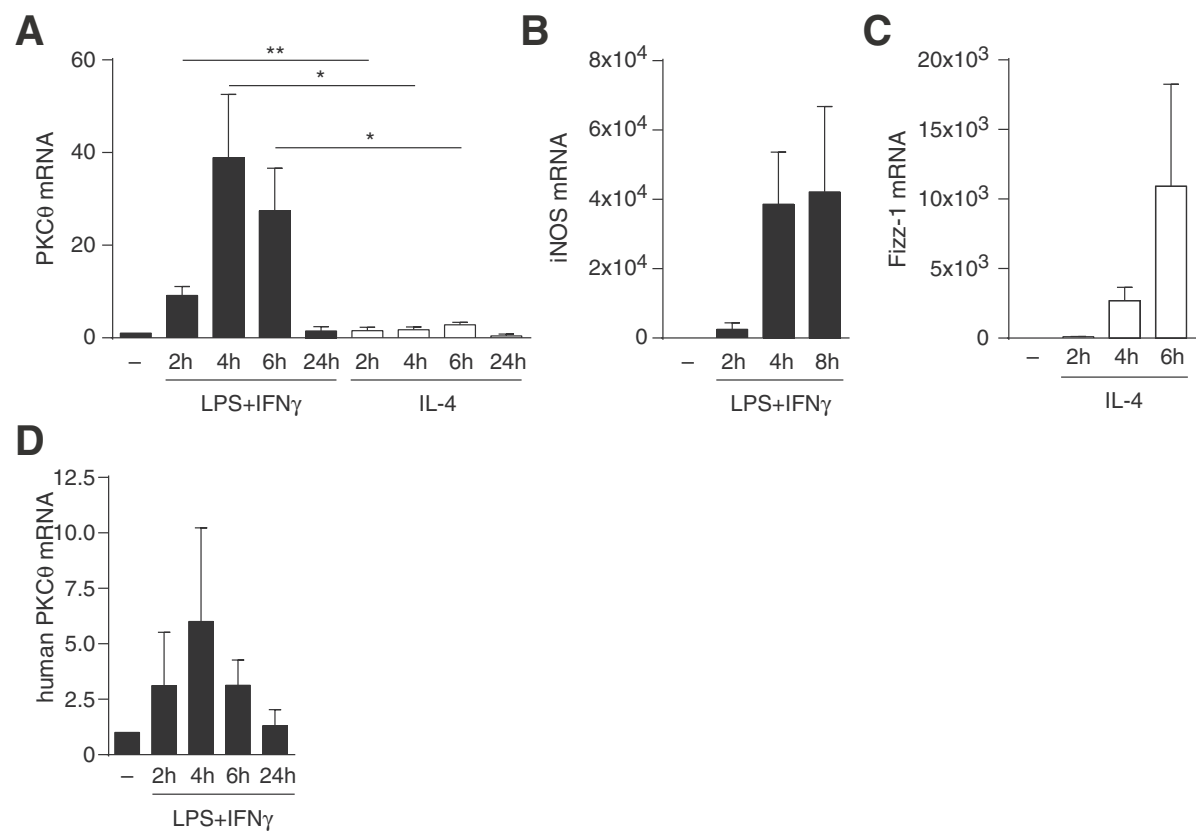

Fig. 1 PKCtheta mRNA levels are transiently induced in an LPS/IFNgamma-dependent manner in both mouse and human macrophages: a BMDMs were differentiated and polarized to CAMs (LPS/IFNgamma) and AAMs (IL-4). PKCtheta gene expression was determined by qRT-PCR after different time points and was found to be selectively expressed under CAM polarization. Data shown are derived from at least three independent experiments and qRT-PCR data were normalized to Gapdh. Error bars denote the mean \pm s.e.m. ${ }^{*} p<0.05 ;{ }^{* *} p<0.01$; Expression of unstimulated macrophages was arbitrarily set to $1 . \mathbf{b}+\mathbf{c}$ In order to control proper polarization of macrophages, gene expression of prominent markers (iNOS for CAM, Fizz-1 for AAM) were validated. $\mathbf{d}$ CD14+ human monocytes from peripheral blood were differentiated and polarized with LPS/IFNgamma. After predetermined time periods, gene expression of PKCtheta was analysed with GRT-PCR, revealing an increase in PKCtheta mRNA levels upon stimulation in the human setting, consistent with results from mouse experiments. Data shown are relative to expression of unstimulated macrophages, which was arbitrarily set to 1. All data were normalized to Gapdh 
IFNgamma-polarized but not in IL-4-polarized cells. Of note, PKCtheta mRNA up-regulation in CAMs peaked at $4 \mathrm{~h}$. These results suggest a subset-selective role for PKCtheta as "signaling intermediate" of pro-inflammatory $\mathrm{M} \phi$. Reminiscent to mouse cells, PKCtheta is inducibly expressed in human CD14+ monocytes polarized with LPS/IFNgamma, indicating a possible role of PKCtheta also in fine-tuning of the cellular phenotype of human cells (Fig. 1d).

\section{PKCtheta is causally involved in the protection from} peritoneal S. typhimurium infection

Based on significant PKCtheta expression level in CAMs, we investigated its potential role in bacterial killing, employing infection assays with intracellular Gramnegative S. typhimurium bacteria in vivo and ex vivo. Evaluation of wild-type and PKCtheta ${ }^{-/-}$mice during systemic infection showed that all of the PKCtheta ${ }^{-/-}$mice died within 16 days whereas a minimum of $50 \%$ wild-type mice survived, suggesting a critical role for PKCtheta in the host defense against S. typhimurium (Fig. 2a). As PKCtheta ${ }^{-1-}$ mice started to die around day 4-5, we monitored the bacterial load in spleen and liver at day 4 which revealed a significant increase in the number of colonyforming units (cfu) of S. typhimurium in PKCtheta ${ }^{-1-}$ mice (Fig. 2b). These data clearly establish an essential and non-redundant positive role of PKCtheta in protection from S. typhimurium.

\section{T cells are not primarily responsible for the reduced} survival of $P K C$ theta ${ }^{-/-}$mice after peritoneal infection with S. typhimurium

To investigate whether $\mathrm{T}$ cells contribute to the significantly reduced survival phenotype of PKCtheta $^{-/-}$mice
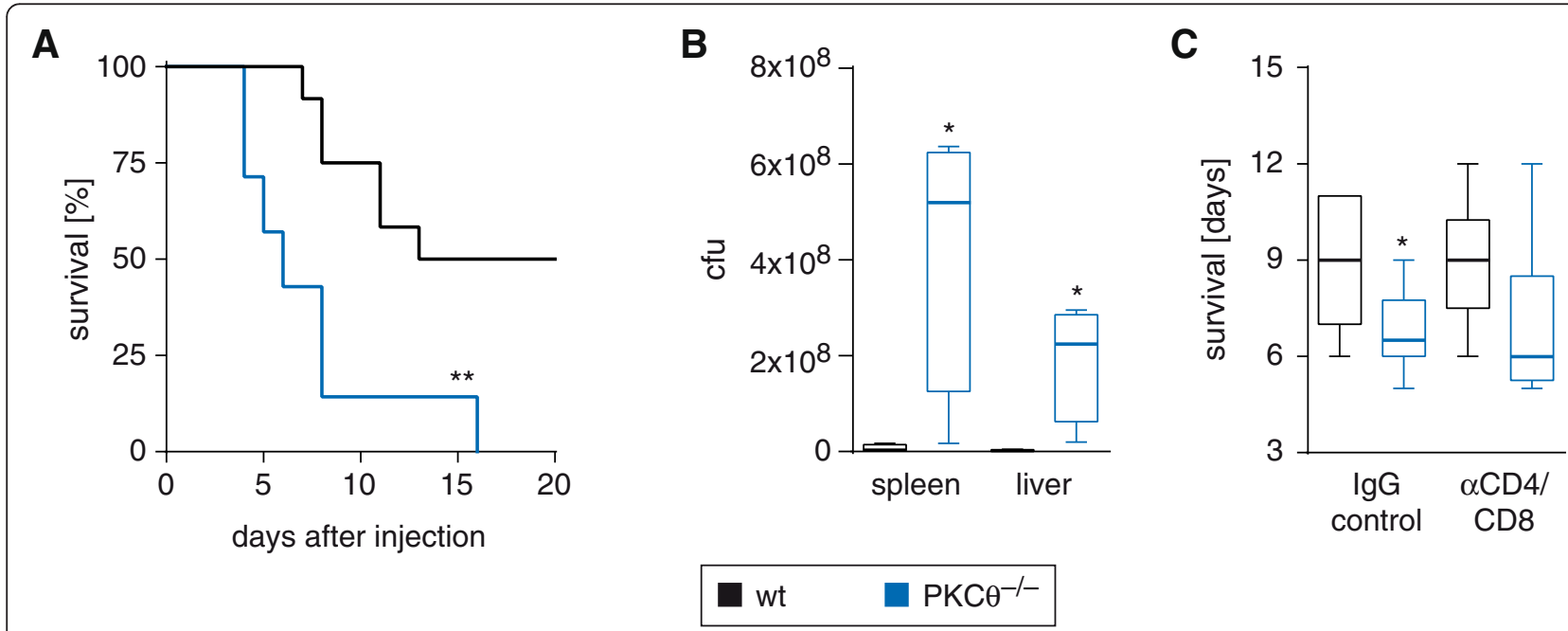

D

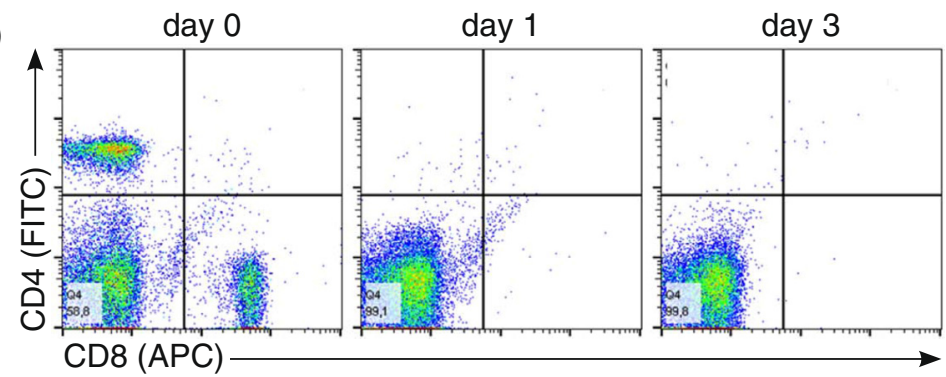

Fig. 2 PKCtheta ${ }^{-1-}$ mice show markedly decreased survival and increased number of colony-forming units in spleen and liver compared to wild-type mice. a Survival studies of wild-type (WT) versus PKCtheta ${ }^{-/-}$mice revealed a significant disadvantage of PKCtheta-deficient animals after intraperitoneal injection of 50,000 cfu of S. typhimurium depicted by a Kaplan-Meier curve and statistically analysed by the log-rank test ( $p=0.0024)$. $\mathbf{b}$ Bacterial loads in spleen and liver were determined on day 4 after infection with $S$. typhimurium and displayed a significant increase in cfu in PKCtheta ${ }^{-/-}$mice. Data are expressed as mean \pm s.e.m. ${ }^{*} p<0.05 ; \mathbf{c}$ In order to investigate the role of T cells in the survival disadvantage of PKCtheta ${ }^{-1-}$ mice after infection with S. typhimurium, CD4 ${ }^{+}$and $\mathrm{CD}^{+} \mathrm{T}$ cells were depleted with the corresponding antibodies prior to infection. IgG isotype antibodies were used for control animals. Results show no difference in survival in T cell-depleted mice versus mice with lgG control, indicating a negligible role of $C D 4^{+}$and $C D 8^{+} T$ cells in the reduced survival rate of PKCtheta ${ }^{-1-}$ mice. Data are expressed as mean \pm s.e.m. ${ }^{*} p<0.05$; $\mathbf{d}$ Depletion of $C D 4^{+}$and $C D 8^{+} T$ cells was controlled by FACS analysis on day 0 (prior to depletion), day 1 (infection with S. typhimurium) and day 3. Antibodies and lgG controls were injected every third day during the course of the experiment 
during S. typhimurium infection, we depleted $C D 4^{+}$and $\mathrm{CD}^{+} \mathrm{T}$ cells with antibodies before injection of S. typhimurium (Fig. 2c). Depletion antibodies were re-injected every 3 days during the course of the experiment. Isotype-matched antibodies were injected as depletion control and effective depletion was controlled by FACS analysis (Fig. 2d). The results showed no significant difference in survival of mice injected either with CD4 and CD8 depletion antibodies or isotype control, indicating that $\mathrm{T}$ cells are not primarily responsible for the reduced survival rate of $P K C$ theta $^{-/-}$mice. These data suggest that PKCtheta ${ }^{-/}$mice fail to mount the appropriate innate immune responses after infection with $S$. typhimurium.

\section{PKCtheta plays a crucial role in repression of IL-10 pro- duction in vitro and in vivo}

In $\mathrm{T}$ cells, PKCtheta is known to affect a wide range of transcription factors such as AP-1, NF-kappaB and NFAT, regulating i.e. IL-2 activation responses. Additionally, PKCtheta influences the transcription of miRNA clusters [18] and, in turn, controls expression of many other genes. To investigate a PKCtheta signaling function upstream of cytokine responses in M $\phi$, LPS/ IFNgamma-polarized BMDMs from wild-type and PKCtheta ${ }^{-/-}$mice were monitored for pro- and antiinflammatory cytokine production at different time points. Although we could not detect any differences in the production of IL-6, IL-12p40, TNF-alpha as well as iNOS expression (data not shown) in polarized PKCtheta ${ }^{-1-}$ CAMs, we found a significantly increased production of the anti-inflammatory cytokine IL-10 in vitro (Fig. 3a). Of, note, the anti-inflammatory cytokine TGF $\beta$ response of polarized PKCtheta ${ }^{-/-}$CAMs, remained unchanged both at the protein and the mRNA levels (Fig. 3b and data not shown), indicating a selectivity of PKCtheta signaling function. Mechanistically and albeit our results do not rule out effects of PKCtheta deficiency on TLR4 signaling responses in general, PKCtheta does not regulate TLR4 signaling leading to IRF3 activation response (Fig. 3c). In agreement with this observation, the significantly lowered survival rates of PKCtheta ${ }^{-1-}$ mice during peritoneal S. typhimurium infection correlated with significantly higher IL-10 serum levels (Fig. 3d). Additionally, after injecting LPS intraperitoneally, the anti-inflammatory IL-10 serum response in PKCtheta-deficient mice was profoundly increased after 2 to $24 \mathrm{~h}$ (Fig. 3e). This further confirms the increased IL-10 production by myeloid cells in another in vivo model. Thus, although $\mathrm{M} \phi$ from either PKCtheta ${ }^{-/-}$or wild-type mice were polarized into CAMs, only wild-type $\mathrm{M} \phi$ but not PKCtheta-deficient $\mathrm{M} \phi$ mounted a functional anti-bacterial activity.
PKCtheta-mediated IL-10 repression affects bacterial killing of macrophages in vitro

Because containment and killing of $S$. typhimurium after invasion of $M \phi$ is a key event in host protection, we monitored intracellular bacterial loads of S. typhimurium-infected $\mathrm{M} \phi$ of both genotypes, reproducibly demonstrating increased bacterial numbers in PKCtheta ${ }^{-/-}$CAMs as compared to wild-type macrophages (Fig. 4a). In order to investigate a direct role of IL-10 hyper-production for the reduced killing capacity of Salmonella-infected PKCtheta ${ }^{-/-}$ $\mathrm{M} \phi$, IL-10 was neutralized with IL-10 blocking- or isotype control antibodies. We observed that IL-10 blocking antibody treatment rescued the killing capacity of PKCthetadeficient cells back to the wild-type level (Fig. 4a). Similar to genetic PKCtheta depletion, pharmacological PKCtheta inhibition by the PKC-selective inhibitor AEB071 (Sotrastaurin) [19] significantly reduced the killing capacity of $S$. typhimurium-infected wild-type $\mathrm{M \phi}$ (Fig. 4b). Taken together, these results suggest that PKCtheta is critically involved in bacterial killing of $S$. typhimurium-infected $\mathrm{M} \phi$ by suppressing IL-10 production.

\section{Discussion}

S. typhimurium is a Gram-negative, motile, facultative intracellular bacterium, which invades and multiplies within mononuclear phagocytic cells in liver, spleen, lymph nodes, and Peyer's plaques [20,21]. S. typhimurium causes severe gastrointestinal disorders in humans and typhoid fever with systemic infections in mice. $M \phi$ functions have been strongly implicated in infectious diseases. $\mathrm{M} \phi$, as one of the first barriers of the innate immune system, rapidly control S. typhimurium. Importantly, however, these bacteria may evade immune control and even multiply within $M \phi$ by mechanisms that are insufficiently understood so far [22, 23].

The immune response to bacterial infection is regulated by the counter-play between different $M \phi$ subsets, namely CAMs and AAMs. Changes in the balance of CAM- and AAM- subsets and their effector cytokines result in alterations of disease progression. Recognition of Gram-negative bacteria in $\mathrm{M} \phi$ involves the binding of LPS to TLR4, leading to secretion of key cytokines [24, 25]. Furthermore, $M \phi$ produce ROS by the phagocyte NADPH oxidase (NOX2; phox) and RNS by the inducible nitric oxide synthase (iNOS; NOS2) to combat bacteria. To counteract chronic pro-inflammatory immune effector mechanisms, the production of antiinflammatory proteins IL-10 and TGFbeta by $M \phi$ is induced [26, 27]. NO, generated by IFNgamma-induced iNOS, is shut down with a shift to Arg1, and YM1 and YM2 are strongly induced, subsequently leading to the induction of a Th2 immune response [28].

IL-10 plays an essential part in dampening inflammation [29]. Consequently, dysregulation of IL-10 is linked with 
A

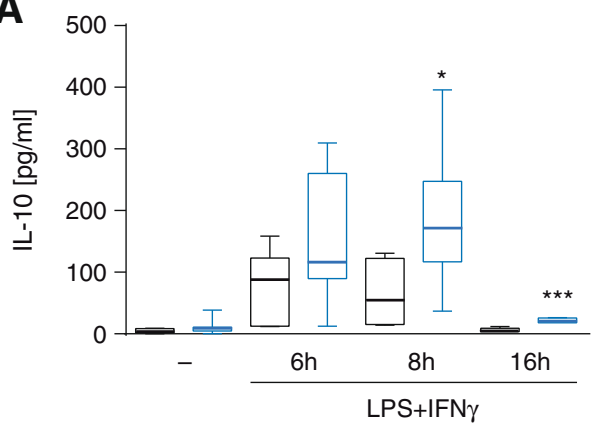

B

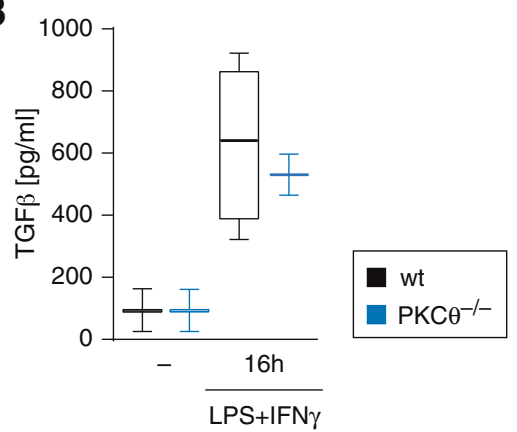

C

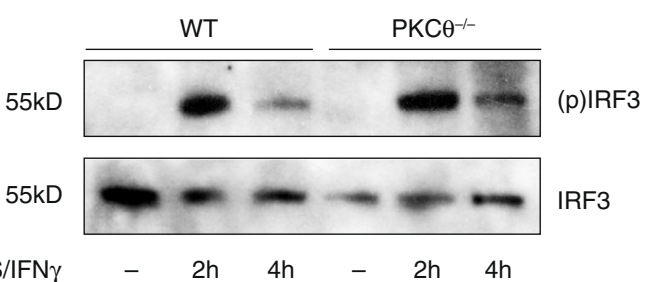

D

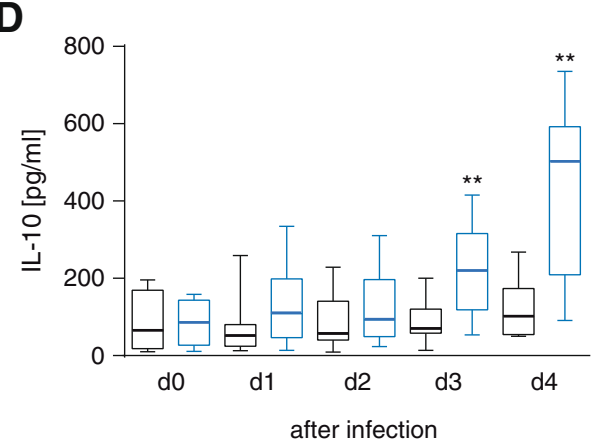

E

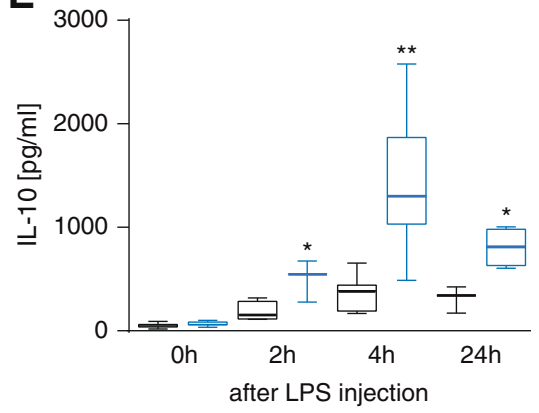

Fig. 3 PKCtheta deficiency leads to IL-10 hyperproduction of bone marrow-derived macrophages. a, b Bioplex technology was used to analyse secretion responses of the anti-inflammatory cytokines IL-10 (a) and TGFbeta (b) in the supernatant of differentiated and LPS/IFNgamma-polarized macrophages from PKCtheta ${ }^{-1-}$ versus WT mice. Error bars show the mean of at least three independent experiments \pm s.e.m. ${ }^{*} p<0.05 ;{ }^{* * *} p<0.001$; c Differentiated BMDM were allowed to rest for $1 \mathrm{~h}$ in X-Vivo 20 medium and were then stimulated with LPS/IFNgamma (100 ng/ml and $10 \mathrm{ng} / \mathrm{ml}$, respectively) for 2 and $4 \mathrm{~h}$ and whole cell lysates were subjected to immunoblotting against phosphorylated IRF3 and total IRF3. $\mathbf{d}$ Analysis of IL-10 serum concentration of WT and PKCtheta ${ }^{-1-}$ mice after infection with S. typhimurium from day 0 to day 4 with Bioplex technology. Error bars show the mean of at least three independent experiments \pm s.e.m. ${ }^{* *} p<0.01$; e IL-10 serum levels from peripheral blood of wild-type featuring PKCtheta ${ }^{-/-}$mice after challenging mice with intraperitoneal LPS injection as an alternative infection model. Data were collected from at least three experiments and shown as mean \pm s.e.m. ${ }^{*} p<0.05 ;{ }^{* *} p<0.01$

susceptibility and an impaired clinical course of numerous infections in mouse models and in humans [30-32]. Mechanistically, distinct signaling and epigenetic chromatin remodelling of the IL1O locus control the production of IL-10. Furthermore, post-transcriptional events through e.g. microRNA represent a critical step for IL-10 transcript stability.

In response to LPS, TLR4 mediated signaling utilizes IRF3 to induce an IL-10 transcription response. Nevertheless, a number of additional transcription factors including signal transducers and activators of transcription (STAT), activator protein (AP), cAMP response element binding protein (CREB), CCATT enhancer/binding protein $(\mathrm{C} / \mathrm{EBP})$, c-musculoaponeurotic fibrosarcoma factor
(c-MAF), and nuclear factor kappa B (NF-kappaB), have been characterized as essential or critical in myeloid cell-type-specific IL-10 gene regulation. Thus a comprehensive understanding of the detailed cellular processes of PKCtheta that contribute in a CAM cellspecific manner to IL-10 transcriptional regulation remains elusive. Albeit we cannot define the exact signaling function of PKCtheta upstream of Il10 transcriptional regulation, the results are in a line with our observation of increased IL-10 formation in PKCtheta-deficient BMDMs and that neutralization of IL-10 results in an improved control of bacterial proliferation that is indistinguishable between wild-type and PKCtheta-deficient macrophages. 


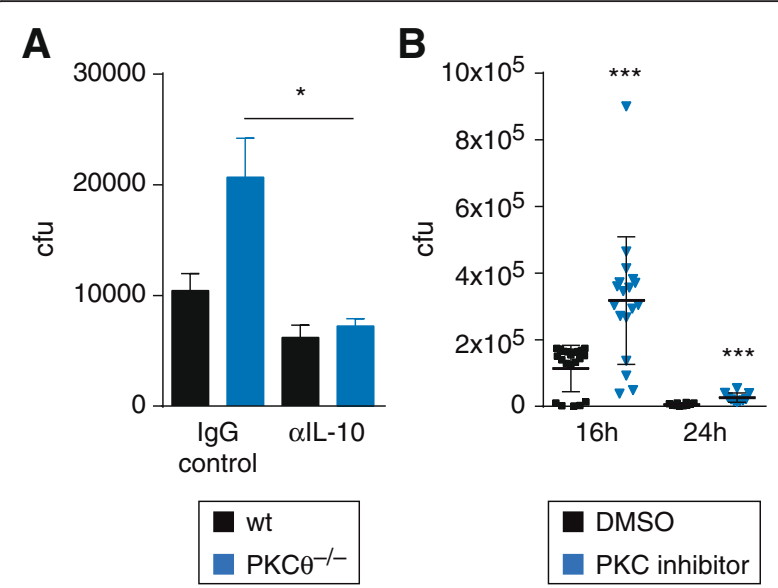

Fig. 4 PKC is involved in bacterial killing of macrophages after infection with S. typhimurium via inhibition of IL-10 deregulation. a Killing capacity of BMDMs from WT versus PKC theta ${ }^{-1-}$ mice was investigated after infection of macrophages with S. typhimurium. Macrophages were treated with IL-10 or lgG control antibodies. Significantly enhanced numbers of colony-forming units (cfu) were found in lysates of macrophages from PKCtheta ${ }^{-/-}$mice compared to WT mice. This effect could be abrogated with IL-10 blockade. Data represent the mean of at least three independent experiments \pm s.e.m. ${ }^{*} p<0.05$; b PKC inhibitor AEB071 enhanced cfu in macrophages infected with $S$. typhimurium highly significantly as shown in a killing experiment with BMDM from WT mice. DMSO served as control vehicle. Error bars denote the mean \pm s.e.m. ${ }^{* * *} p<0.001$

\section{Conclusions}

We demonstrate that PKCtheta contributes to a hostprotective immune response against peritoneal $S$. typhimurium infection by its selective involvement during early (up to $24 \mathrm{~h}$ ) pro-inflammatory CAM polarization. This time frame is in agreement with the fact that in these cells PKCtheta is induced immediately and transiently after LPS/IFNgamma engagement. PKCtheta deficiency, as defined in this study, leads to an altered CAM phenotype, based on an incomplete suppression of the macrophage deactivating cytokine IL-10. The detailed molecular basis of PKCtheta-dependent regulation of IL-10 production under CAM-promoting conditions, and thus the phenotypic instability of PKCtheta ${ }^{-/}$CAMs, however, remains unknown. Nevertheless, the data define a previously unknown regulatory role of PKCtheta in macrophage driven innate defense mechanisms against bacterial infections. As an innovative paradigm, CAM-intrinsic PKCtheta may represent a transiently inducible signaling intermediate leading to IL-10 suppression, and subsequently, to an effective anti-bacterial immunity against intracellular bacteria such as S. typhimurium. This hypothesis is based on the increased IL-10 expression observed during the PKCtheta $^{-/}$CAM-priming phase. Of note, this deregulated IL-10 expression level observed in PKCtheta ${ }^{-/-}$ CAMs is directly responsible for their bacterial killing defects after infection with $S$. typhimurium.
In summary, the findings of this study provide genetic evidence that PKCtheta is an early factor involved in developing a stable CAM-mediated immune response in vitro and in vivo. Our results provide first experimental evidence for an involvement of PKCtheta in $\mathrm{M} \phi$ biology, a finding relevant for the understanding of PKCtheta in innate antimicrobial immune effector function.

\section{Methods}

\section{Mice}

Wild-type and PKCtheta ${ }^{-/-}$mice (on mixed C57BL/ 6x129/Sv background) were maintained under specific pathogen-free conditions in the central animal facility of the Medical University of Innsbruck. PKCtheta ${ }^{-1-}$ mice have previously been described in detail (3). All animal experiments have been performed in accordance with national and European guidelines and have been reviewed and authorized by the committee on animal experiments (Federal Ministry of Science, Research and Economy-66.01l/0128-WF/V/3b/2014).

\section{Cell culture}

Bone marrow-derived macrophages (BMDM) were harvested from tibiae and femora of 8 to 12-week-old mice and differentiated for 7 days in complete DMEM (Biochrom) supplemented with 10 \% FCS, 2 mM L-glutamine, $10,000 \mathrm{U} / \mathrm{mL}$ penicillin plus $10 \mathrm{mg} / \mathrm{mL}$ streptomycin (all from Biochrom) and 15 \% L929 supernatant. Medium was replaced after 4 days. On day 7 , cells were washed with phosphate-buffered saline (PBS) and polarized with either $10 \mathrm{ng} / \mathrm{ml}$ LPS (L6511; Sigma-Aldrich) and $10 \mathrm{ng} / \mathrm{ml}$ IFNgamma (BMS326, eBioscience) or with $10 \mathrm{ng} / \mathrm{ml} \mathrm{IL-4}$ (14-8041-62; eBioscience) for different time periods in XVivo 20 medium (BE04-448Q, Lonza).

\section{Western blotting}

BMDM $\left(1 \times 10^{6}\right)$ were lysed in lysis buffer (50 mM Tris$\mathrm{HCl}, \mathrm{pH}$ 7.3, $5 \mathrm{mM} \mathrm{NaF}, 5 \mathrm{mM} \mathrm{Na}_{3} \mathrm{VO}_{4}, 5 \mathrm{mM} \mathrm{NaP}{ }_{2} \mathrm{P}$, $5 \mathrm{mM}$ EDTA, $50 \mathrm{mM} \mathrm{NaCl}, 1 \% \mathrm{NP}-40,50 \mu \mathrm{g} / \mathrm{ml}$ aprotinin, $50 \mu \mathrm{g} / \mathrm{ml}$ leupeptin) and subjected to SDS-PAGE on Bis/Tris-buffered gels (Novex). After transfer to nitrocellulose membrane by semi-dry blotting, Ser-396 phosphorylated as well as pan-IRF3 were detected by immune blotting (both with antibodies from Cell Signaling Technology).

\section{Gene expression analysis}

Total RNA was isolated using the RNeasy ${ }^{\circledR}$ Mini Kit (Qiagen) according to the manufacturer's instructions. cDNA was synthesized using the Qiagen Omniscript RT Kit and oligo(dT) primers (Promega). Expression analysis was performed with real-time PCR using TaqMan technology (assays from Applied Biosystem). Reactions were run with Applied Biosystems 7500 Fast Sequence 
Detection System. All gene expressions were normalized to Gapdh.

\section{Human CD14+ monocyte polarization}

Ficoll-Paque Premium (17-5442-02; GE-Life Sciences) was used to isolate mononuclear cells from blood of healthy volunteers according to the manufacturer's instructions. CD14+ cells were positively selected with human CD14 MicroBeads (130-050-201; MACS Miltenyi Biotec) and cultured in DMEM (BioWhittaker BE12-707 F, Lonza) supplemented with $10 \%$ FCS, 2 mM L-glutamine, $1 \%$ penicillin plus streptomycin $(10,000 \mathrm{U} / \mathrm{mL}$ penicillin and $10 \mathrm{mg} / \mathrm{mL}$ streptomycin in $0.9 \% \mathrm{NaCl}$ ), and $100 \mathrm{ng} / \mathrm{ml}$ of human GM-CSF (572903, BioLegend). Cells were fed on day 4 and stimulated with $10 \mathrm{ng} / \mathrm{ml}$ LPS (lipopolysaccharide from S. typhimurium L6511; Sigma-Aldrich, Vienna, Austria) and $10 \mathrm{ng} / \mathrm{ml}$ human IFNgamma (570204, BioLegend) on day 7 for the indicated time periods.

\section{Bacteria-induced infection model}

8 to 12-week-old male WT and PKCtheta ${ }^{-1-}$ mice were infected intraperitoneally with 50,000 cfu of Salmonella enterica serovar typhimurium (ATCC14028) diluted in $200 \mu \mathrm{l}$ PBS, and survival was monitored over 21 days. For cytokine measurement, blood samples were taken on day 0 to day 4 and serum was collected. Bacterial load of organs was determined by plating serial dilutions of organ homogenates from day 4 after infection on LB broth agar (L7275-500TAB; Sigma-Aldrich) under sterile conditions and the number of bacteria was calculated per gram of tissue after cultivation.

\section{In vivo $\mathrm{T}$ cell depletion}

Mice were injected with $500 \mu \mathrm{g}$ of anti-mouse CD4 (clone GK1.5; BE003-1) and anti-mouse CD8 antibody (clone YTS 169.4; BE0117), or the corresponding IgG2b (clone LTF-2; BE0090) control (all from BioXCell, USA) 1 day prior to infection with S. typhimurium. Facial vein blood was taken after $24 \mathrm{~h}$ to control $\mathrm{T}$ cell depletion by FACS analysis using antiCD4-FITC, antiCD8-APC, and antiCD3PE (all from eBioscience). Thereafter, 5,000 to 30,000 cfu $S$. typhimurium were injected intraperitoneally. $300 \mu \mathrm{g}$ of depletion antibodies or isotype control were administered every third day throughout the entire experiment.

\section{Cytokine measurements}

IL-10 and TGFbeta cytokine levels from from serum or cell culture supernatants were analyzed with Bio-Plex multianalyte technology (BioRad).

\section{Endotoxin-induced infection model}

WT and PKCtheta ${ }^{-1-}$ mice were treated with intraperitoneal injection of $15 \mathrm{mg}$ LPS (lipopolysaccharide from $S$. typhimurium L6511; Sigma-Aldrich) per $\mathrm{kg}$ of body weight. Serum was collected $4 \mathrm{~h}$ after LPS challenge for analysis of IL-10.

\section{Salmonella infection in vitro}

Macrophages were isolated as described above and incubated in complete DMEM without antibiotics. Wild-type strain S. typhimurium was cultured in LB broth to latelogarithmic phase. $\mathrm{M} \phi$ were infected with $S$. typhimurium at a multiplicity of infection (MOI) of 10 for $1 \mathrm{~h}$. Thereafter, cells were washed with PBS and incubated in complete DMEM containing gentamycin (Gibco). For killing experiments, prior to infection, cells were treated with a monoclonal rat anti-mouse IL-10 antibody (10 $\mathrm{\mu g} / \mathrm{ml}$; clone JES5-2A5; 504903; BioLegend) or the appropriate isotype control $(10 \mu \mathrm{g} / \mathrm{ml}$; clone RTK2071; 400413; BioLegend) for $23 \mathrm{~h}$. AEB071 (sotrastaurin) was used as PKC inhibitor in killing experiments and cells were treated with $1 \mu \mathrm{M}$ for $23 \mathrm{~h}$. After infection, intracellular bacterial loads were harvested with $0.5 \%$ sodium deoxycholic acid (D6750; Sigma-Aldrich) as described previously [33].

\section{Abbreviations}

AAM, alternatively activated macrophages; CAM, classically activated macrophages; LPS, lipopolysaccharide; M $\varphi$, macrophages; PKC, Protein kinase C; S., salmonella

\section{Acknowledgements}

The authors want to thank Hermann Dietrich for the cooperation with the central laboratory animal facility and Nina Posch for help with western blotting.

\section{Funding}

This work was supported by grants from the FWF Austrian Science Fund (25044-B21 to GB, M1636-B23 to KS \& GB, TRP-188 to GW) and by the intramural funding program of the Medical University Innsbruck for young scientists MUI-START, Project 2013042005 (to CPO).

\section{Availability of data and materials} Not applicable.

\section{Authors' contributions}

CPO designed and performed the animal experiments, participated in the experimental studies and drafted the manuscript. KAS performed parts of the animal experiments, molecular genetic studies and experiments during revision and helped drafting the revised manuscript. SP carried out parts of the animal and cellular studies. MN conceived and designed the animal experiments. KS contributed to the molecular genetic experiments. VK performed the FACS analysis. DH helped with the human monocyte experiments. NT participated in study design. NHK participated in study design and helped with statistical analysis. TG participated in study design. GW participated in study design and analysed parts of the research. GB coordinated the project and wrote the manuscript. All authors read and approved the final manuscript.

\section{Competing interests}

The authors declare that they have no competing interests.

\section{Consent for publication}

All authors have given their consent for publication.

Ethics approval and consent to participate Not applicable. 


\section{Author details}

'Department for Pharmacology and Genetics, Division of Translational Cell Genetics, Peter Mayr Straße 1a, 6020 Innsbruck, Austria. ${ }^{2}$ Department of Internal Medicine VI/Infectious Diseases, Immunology, Rheumatology, Pneumology, Medical University Innsbruck, Anichstraße 35, 6020 Innsbruck, Austria.

Received: 5 April 2016 Accepted: 22 July 2016

Published online: 28 July 2016

\section{References}

1. Marsland BJ, Soos TJ, Spath G, Littman DR, Kopf M. Protein kinase C theta is critical for the development of in vivo Th2 cell but not Th1 cell responses. J Exp Med. 2004;200(2):181-9.

2. Salek-Ardakani S, So T, Halteman BS, Altman A, Croft M. Differential regulation of Th2 and Th1 lung inflammatory responses by protein kinase $C$ theta. J Immunol. 2004;173(10):6440-7.

3. Salek-Ardakani S, So T, Halteman BS, Altman A, Croft M. Protein kinase Ctheta controls Th1 cells in experimental autoimmune encephalomyelitis. J Immunol. 2005;175(11):7635-41.

4. Paulson JC. Innate immune response triggers lupus-like autoimmune disease Cell. 2007;130(4):589-91.

5. Reese TA, Liang HE, Tager AM, Luster AD, Van Rooijen N, Voehringer D, et al. Chitin induces accumulation in tissue of innate immune cells associated with allergy. Nature. 2007:447(7140):92-6.

6. Munitz A, Waddell A, Seidu L, Cole ET, Ahrens R, Hogan SP, et al. Resistin-like molecule alpha enhances myeloid cell activation and promotes colitis. J Allergy Clin Immunol. 2008;122(6):1200-7. e1.

7. Gordon S, Martinez FO. Alternative activation of macrophages: mechanism and functions. Immunity. 2010;32(5):593-604.

8. Hallam S, Escorcio-Correia M, Soper R, Schultheiss A, Hagemann T. Activated macrophages in the tumour microenvironment-dancing to the tune of TLR and NF-kappaB. J Pathol. 2009:219(2):143-52.

9. Sun Z, Arendt CW, Ellmeier W, Schaeffer EM, Sunshine MJ, Gandhi L, et al. PKCtheta is required for TCR-induced NF-kappaB activation in mature but not immature T lymphocytes. Nature. 2000;404(6776):402-7.

10. Pfeifhofer C, Kofler K, Gruber T, Tabrizi NG, Lutz C, Maly K, et al. PKCtheta affects $\mathrm{Ca}^{2+}$ mobilization and NFAT activation in primary mouse T cells. J Exp Med. 2003:197(11):1525-35.

11. Madaro L, Pelle A, Nicoletti C, Crupi A, Marrocco V, Bossi G, et al. PKCtheta ablation improves healing in a mouse model of muscular dystrophy. PLoS One. 2012;7(2):e31515

12. $M a$ AZ, Zhang $Q$, Song ZY. TNFalpha alter cholesterol metabolism in human macrophages via PKCtheta-dependent pathway. BMC Biochem. 2013;14:20.

13. von Knethen A, Tautenhahn A, Link H, Lindemann D, Brune B. Activation-induced depletion of PKCalpha provokes desensitization of monocytes/macrophages in sepsis. J Immunol. 2005;174(8):4960-5.

14. Shin DM, Yang CS, Lee JY, Lee SJ, Choi HH, Lee HM, et al. Mycobacterium tuberculosis lipoprotein-induced association of TLR2 with protein kinase C zeta in lipid rafts contributes to reactive oxygen species-dependent inflammatory signaling in macrophages. Cell Microbiol. 2008;10(9):1893-905

15. Leppanen T, Korhonen R, Laavola M, Nieminen R, Tuominen RK, Moilanen E. Down-regulation of protein kinase Cdelta inhibits inducible nitric oxide synthase expression through IRF1. PLoS One. 2013;8(1):e52741.

16. Hardy PO, Diallo TO, Matte C, Descoteaux A. Roles of phosphatidylinositol 3-kinase and p38 mitogen-activated protein kinase in the regulation of protein kinase C-alpha activation in interferon-gamma-stimulated macrophages. Immunology. 2009;128(1 Suppl):e652-60.

17. Brezar V, Tu WJ, Seddiki N. PKCtheta in Regulatory and Effector T-cell Functions. Front Immunol. 2015:6:530.

18. Sutcliffe EL, Bunting KL, He YQ, Li J, Phetsouphanh C, Seddiki N, et al. Chromatin-associated protein kinase C-theta regulates an inducible gene expression program and microRNAs in human T lymphocytes. Mol Cell. 2011;41(6):704-19.

19. Evenou JP, Wagner J, Zenke G, Brinkmann V, Wagner K, Kovarik J, et al. The potent protein kinase C-selective inhibitor AEB071 (sotrastaurin) represents a new class of immunosuppressive agents affecting early T-cell activation. J Pharmacol Exp Ther. 2009;330(3):792-801.

20. Hsu HS. Pathogenesis and immunity in murine salmonellosis. Microbiol Rev. 1989;53(4):390-409.
21. Richter-Dahlfors A, Buchan AM, Finlay BB. Murine salmonellosis studied by confocal microscopy: Salmonella typhimurium resides intracellularly inside macrophages and exerts a cytotoxic effect on phagocytes in vivo. J Exp Med. 1997:186(4):569-80.

22. Weiss G, Schaible UE. Macrophage defense mechanisms against intracellular bacteria. Immunol Rev. 2015;264(1):182-203.

23. Behnsen J, Perez-Lopez A, Nuccio SP, Raffatellu M. Exploiting host immunity: the Salmonella paradigm. Trends Immunol. 2015;36(2):112-20.

24. Nagai Y, Akashi S, Nagafuku M, Ogata M, Iwakura Y, Akira S, et al. Essential role of MD-2 in LPS responsiveness and TLR4 distribution. Nat Immunol. 2002:3(7):667-72.

25. Bode JG, Ehlting C, Haussinger D. The macrophage response towards LPS and its control through the p38(MAPK)-STAT3 axis. Cell Signal. 2012;24(6): 1185-94.

26. Moro K, Yamada T, Tanabe M, Takeuchi T, Ikawa T, Kawamoto H, et al. Innate production of $\mathrm{T}(\mathrm{H}) 2$ cytokines by adipose tissue-associated c-Kit(+)Sca-1(+) lymphoid cells. Nature. 2010:463(7280):540-4.

27. Neill DR, Wong SH, Bellosi A, Flynn RJ, Daly M, Langford TK, et al. Nuocytes represent a new innate effector leukocyte that mediates type-2 immunity. Nature. 2010:464(7293):1367-70.

28. El Kasmi KC, Qualls JE, Pesce JT, Smith AM, Thompson RW, Henao-Tamayo $M$, et al. Toll-like receptor-induced arginase 1 in macrophages thwarts effective immunity against intracellular pathogens. Nat Immunol. 2008;9(12): 1399-406.

29. Sabat R, Grutz G, Warszawska K, Kirsch S, Witte E, Wolk K, et al. Biology of interleukin-10. Cytokine Growth Factor Rev. 2010;21(5):331-44.

30. Li C, Corraliza I, Langhorne J. A defect in interleukin-10 leads to enhanced malarial disease in Plasmodium chabaudi infection in mice. Infect Immun 1999;67(9):4435-42.

31. O'Garra A, Barrat FJ, Castro AG, Vicari A, Hawrylowicz C. Strategies for use of IL-10 or its antagonists in human disease. Immunol Rev. 2008;223:114-31.

32. Warszawska JM, Gawish R, Sharif O, Sigel S, Doninger B, Lakovits K, et al. Lipocalin 2 deactivates macrophages and worsens pneumococcal pneumonia outcomes. J Clin Invest. 2013;123(8):3363-72.

33. Nairz M, Theurl I, Schroll A, Theurl M, Fritsche G, Lindner $E$, et al. Absence of functional Hfe protects mice from invasive Salmonella enterica serovar Typhimurium infection via induction of lipocalin-2. Blood. 2009;114(17):3642-51.

\section{Submit your next manuscript to BioMed Central and we will help you at every step:}

- We accept pre-submission inquiries

- Our selector tool helps you to find the most relevant journal

- We provide round the clock customer support

- Convenient online submission

- Thorough peer review

- Inclusion in PubMed and all major indexing services

- Maximum visibility for your research

Submit your manuscript at www.biomedcentral.com/submit 